Over- and undernutrition: challenges and approaches. 29 June-2 July 2009

\title{
The MEtabolomics to characterise Dietary Exposure (MEDE) Study: kinetics of metabolite responses to a test breakfast
}

\author{
G. Fave', S. Zhou ${ }^{2}$, M. E. Beckmann², G. Harold ${ }^{1}$, W. Linn $^{2}$, K. N. Tailliart ${ }^{2}$, J. H. Draper ${ }^{2}$ \\ and J. C. Mathers 1 \\ ${ }^{1}$ Human Nutrition Research Centre, School of Clinical Medical Sciences, Newcastle University, Newcastle upon Tyne \\ NE2 4HH, UK and ${ }^{2}$ Institute of Biological Sciences, Aberystwyth University, Aberystwyth, Ceredigion SY23 3DA, UK
}

Measurement of dietary exposure is an essential component of much health-related research. However, conventional tools for collecting information on what foods have been eaten and in what amounts (e.g. diet diaries, FFQ and $24 \mathrm{~h}$ recalls) are subject to participant bias and depend on food composition tables ${ }^{(1)}$. The MEDE Study has been designed to provide proof of principle that metabolomics could be a modern non-subjective approach to characterise dietary exposure ${ }^{(2)}$ without the need for diaries and questionnaires.

The first phase of the MEDE Study aimed to develop experimental protocols to measure the metabolite profiles in biofluids before and after consumption of a specific test meal. Fasting and postprandial metabolite profiles and the kinetics of metabolite signals were investigated. Under controlled conditions, twelve healthy non-smoking adults (three post-menopausal women and nine men) consumed a carefully-designed test breakfast (orange juice, tea, butter croissant and cornflakes with milk) on two occasions (visits A and B). Blood, urine and saliva samples were collected in the fasted state and at 2, 4, 6 and $8 \mathrm{~h}$ after meal consumption. After collection of the $4 \mathrm{~h}$ samples subjects were provided with a 'metabolomically neutral' light lunch (white bread, poached eggs and mineral water). Metabolites in the biofluids were analysed by flow injection electrospray (FIE)-MS fingerprinting and GC-time of flight-MS profiling followed by multivariate data analysis. Serum and urine were also analysed for nutritional and inflammatory status markers. To investigate potential contributors to inter-individual metabolome differences, anthropometric characteristics (BMI, waist and hip circumferences, body fat content), habitual diet (FFQ), physical activity (ActiGraph; ActiGraph, Pensacola, FL, USA) and feelings of satiety in response to the test meal (visual analogue scales) were measured.

Linear discriminant analysis (LDA) by sample class of both urine and plasma samples showed a clear discrimination between fasting samples and any of the fed samples (Eigenvalues of 5.8 and 6.7 for urine and plasma samples respectively with FIE-MS fingerprinting). LDA by individual per visit of plasma samples showed a very satisfactory grouping of both visit A and B for each volunteer. There were dynamic changes in metabolite patterns from before breakfast to well after the light lunch with larger-than-expected effects of the 'neutral' light lunch.

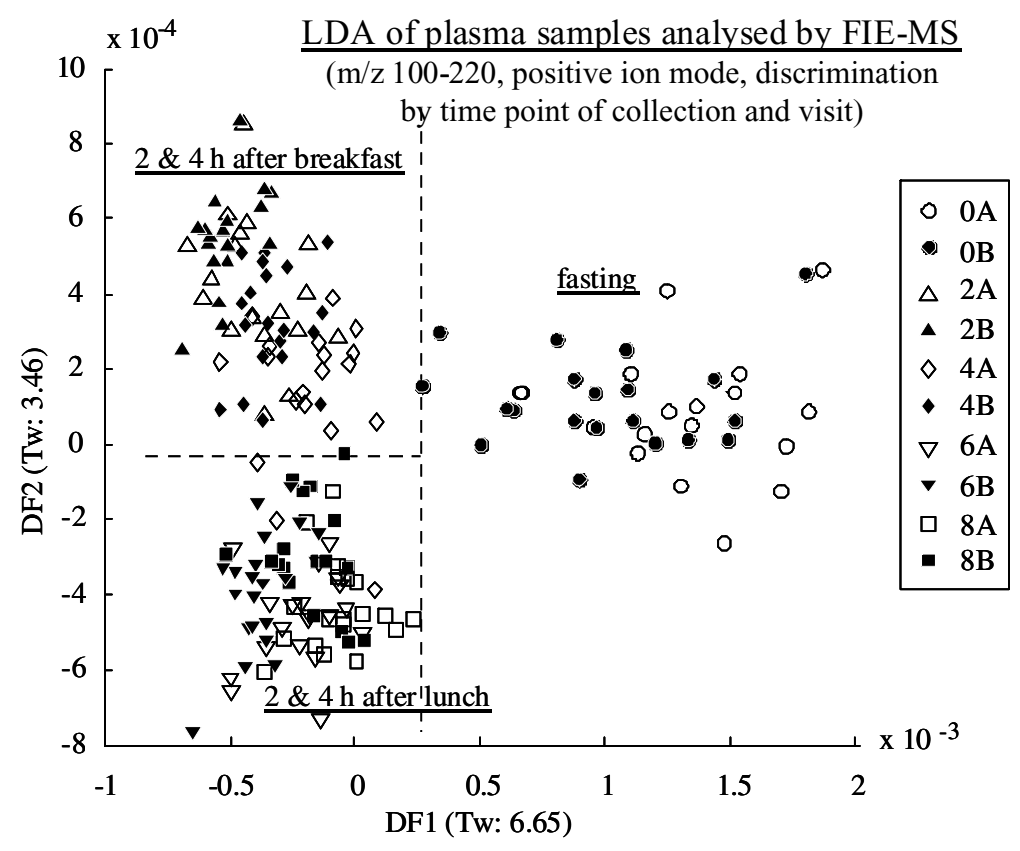

Phase 1 of the MEDE Study has established reproducible protocols to determine metabolome profiles in the fasting state and determined the kinetics of response to a test breakfast. This outcome provides a solid platform to investigate the metabolome signals responsible for discrimination between food exposures and to develop novel exposure biomarkers for specific foods.

This research project is supported by the UK Food Standard Agency (Project N05073). The authors thank Claire Kent, Heather E. Gifford, Julie Coaker and Linda Penn for their practical support.

1. Bingham SA (2002) Public Health Nutr 5, 821-827.

2. Gibney MJ, Walsh M, Brennan L et al. (2005) Am J Clin Nutr 82, 497-503. 